Provided for non-commercial research and education use. Not for reproduction, distribution or commercial use.

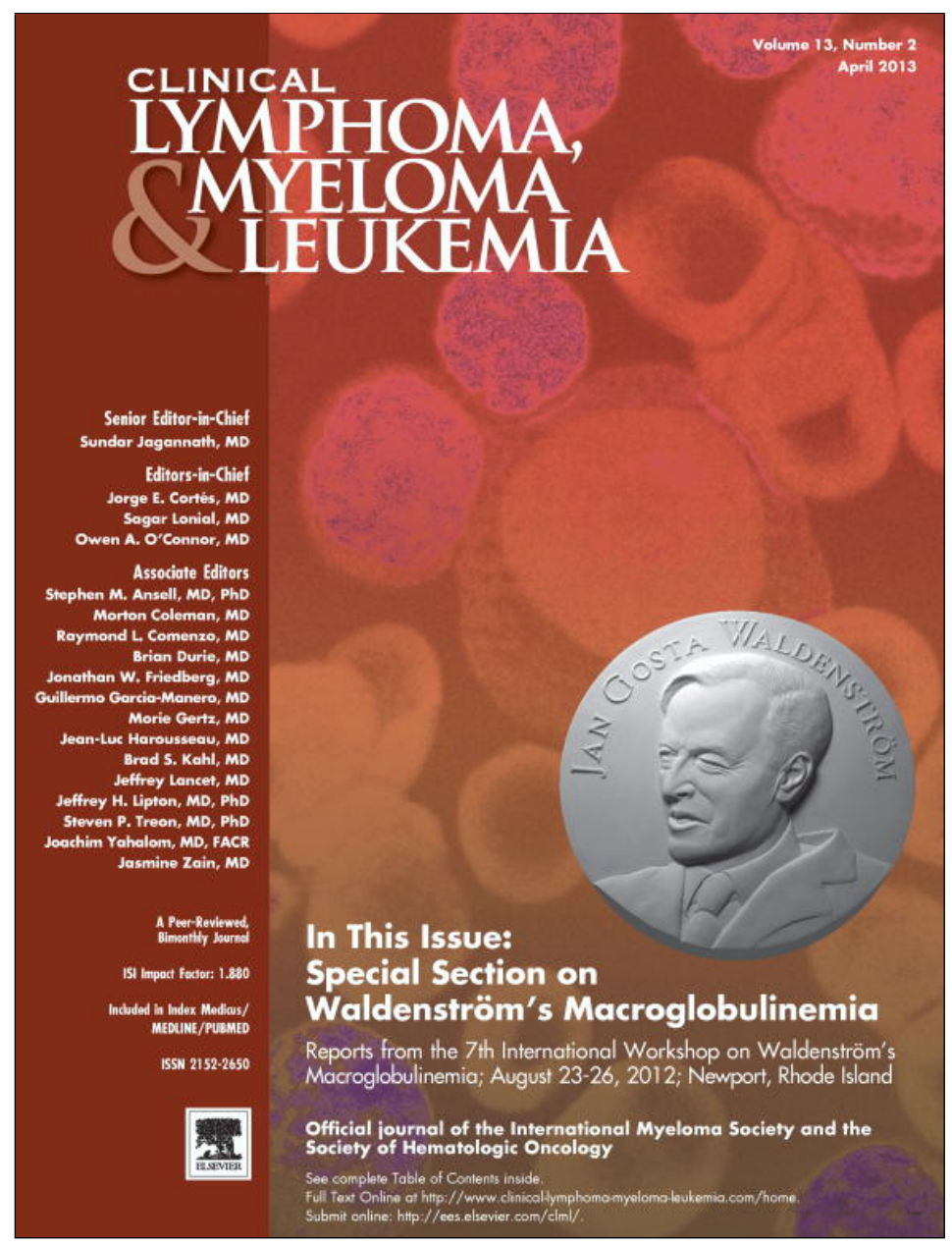

This article appeared in a journal published by Elsevier. The attached copy is furnished to the author for internal non-commercial research and education use, including for instruction at the authors institution and sharing with colleagues.

Other uses, including reproduction and distribution, or selling or licensing copies, or posting to personal, institutional or third party websites are prohibited.

In most cases authors are permitted to post their version of the article (e.g. in Word or Tex form) to their personal website or institutional repository. Authors requiring further information regarding Elsevier's archiving and manuscript policies are encouraged to visit:

http://www.elsevier.com/authorsrights 


\title{
Targeting the Toll-like Receptor/Interleukin 1 Receptor Pathway in Human Diseases: Rational Design of MyD88 Inhibitors
}

\author{
Maria Loiarro, ${ }^{1,2}$ Vito Ruggiero, ${ }^{3}$ Claudio Sette ${ }^{1,2}$ \\ Clinical Lymphoma, Myeloma \& Leukemia, Vol. 13, No. 2, 222-6 @ 2013 Elsevier Inc. All rights reserved. \\ Keywords: Human diseases, MyD88, Signaling, Targeting, Toll-like receptor/interleukin 1 receptor
}

\begin{abstract}
Toll-like receptor (TLR)/interleukin (IL) 1 receptor (IL-1R) play a fundamental role in the immune response. These receptors are distributed in various cellular compartments and recognize different components of pathogens. All TLR/IL-1Rs, with the exception of TLR3, interact with MyD88, an intracellular adapter protein that triggers a signaling cascade that culminates in the expression of inflammatory genes. Because aberrant activation of TLR/IL-1Rs can promote the onset of inflammatory or autoimmune diseases and malignancies, this pathway has attracted considerable interest as a potential therapeutic target. Given the central role of MyD88 in TLR/IL-1R signaling, we set out different strategies to develop drugs that can block its function. Structural and functional analysis of the MyD88 domains allowed us to identify crucial residues required for MyD88 homodimerization. Moreover, we developed small cell-permeable peptides and peptidomimetic agents that inhibit MyD88 homodimerization and function. Our results pave the way for the development of new therapeutic drugs for the inhibition of MyD88dependent signaling.
\end{abstract}

\section{Introduction}

Toll-like receptors (TLR)/interleukin (IL)-1 receptors (IL-1R) play key roles in host defense and inflammation. TLR/IL-1Rs are expressed by a variety of immune and nonimmune cells ${ }^{1}$ and recognize exogenous pathogen-associated molecular patterns, such as lipopolysaccharide, lipopeptides, flagellin, bacterial DNA, and viral double-stranded RNA, as well as endogenous, host-derived dangerassociated molecular patterns, as high mobility group Box 1 and heat shock proteins. ${ }^{2}$ TLR/IL-1Rs are characterized by the presence of the Toll/IL-1 receptor (TIR) domain in their cytoplasmic portion. ${ }^{3}$ The

${ }^{1}$ Department of Biomedicine, Prevention, University of Rome "Tor Vergata," Rome, Italy

${ }^{2}$ Laboratory of Neuroembryology, Fondazione Santa Lucia, Rome, Italy

${ }^{3}$ Research and Development, Sigma-Tau, S.p.A., Pomezia (RM), Italy

Address for correspondence: Claudio Sette, PhD, Department of Biomedicine, Prevention, University of Rome "Tor Vergata," 00133, Rome, Italy E-mail contact: claudio.sette@uniroma2.it
TIR domain is required for the recruitment of adapter proteins through interaction with their own TIR domain. The recruitment of these adapters then triggers the pathway involved in signal transduction. ${ }^{4}$ With the sole exception of TLR3, all TLR/IL-1Rs use MyD88 as adapter protein, either alone or in association with other adapters. ${ }^{4}$ MyD88 has a modular structure that consists of an N-terminal death domain (DD) and a C-terminal TIR domain, separated by a short linker sequence. ${ }^{5}$ Upon ligand recognition, MyD88 is recruited as a dimer to the cytoplasmic domain of TLR/IL-1Rs via homophilic interaction between the respective TIR domains. This, in turn, allows MyD88 to associate with members of the IL-1R-associated kinase (IRAK) family through homophilic interaction between the respective DDs. This multimeric complex, named Myddosome, propagates the signal downstream of the receptors, with consequent activation of MAP kinases and the transcription factors nuclear factor-kappa B (NF- $\kappa$ B), activator protein-1 (AP-1), interferon regulatory factors (IRFs), to elicit antipathogen responses and inflammation. ${ }^{6,7}$

Dysregulation of the TLR/IL-1R pathway was suggested to promote the onset of various autoimmune, chronic inflammatory, and infectious diseases, ${ }^{8}$ and it also was recently involved in tumorigenesis. ${ }^{9}{ }^{10}$ Notably, MyD88 itself was shown to play a determinant role in the promotion of several human cancers, including blood malignancies such as lymphoma and chronic lymphocytic leukemia. ${ }^{11-15}$ For these reasons, the TLR/IL-1R pathway has attracted considerable interest as a potential therapeutic target. ${ }^{16,17}$

\section{Rational Development of MyD88 Inhibitors}

Given its central role in the TLR/IL-1R signaling pathway, interfering with the function of $\mathrm{MyD} 88$ is likely to represent a suitable approach for therapeutic treatment of diseases in which these receptors are involved. MyD88 has no catalytic activity, but its ability to homodimerize and to interact with upstream receptors and downstream kinases is crucial for its function. Thus, it is conceivable that MyD88 activity can be inhibited at various steps involved in the formation of the Myddosome complex, such as homodimerization of 
Figure 1 Targeting the Toll/Interleukin (IL)-1 Receptor (TIR) TIR or Death Domains (DD) of MyD88 for the Development of Toll-like Receptor (TLR)/IL-1R Signaling Pathway Inhibitors. TLR/LL-1Rs Recognize Ligands With Their Extracellular Portion Constituted of Leucine-Rich Repeat Domain (LRR) or Immunoglobulin-like Domains, Respectively. Upon Ligand Stimulation, They Interact With MyD88 by Their Respective TIR Domains. MyD88 Oligomerizes and Recruits the IL-1R-associated Kinase (IRAK) by Their Respective DDs. This Multimeric Complex Propagates the Signal Downstream of the Receptors, With Consequent Activation of Nuclear Factor-kappa B (NF- $\kappa$ B) and Induction of Genes Involved in Inflammatory Responses. Inhibitors of the Homodimerization of the TIR Domain (A) or DD (B) of MyD88 Can Interfere With the Interaction Between TLR/LL-1Rs and MyD88 (A) or the Recruitment of IRAKs by MyD88 (B), Thereby Preventing the Activation of NF- $\kappa$ B
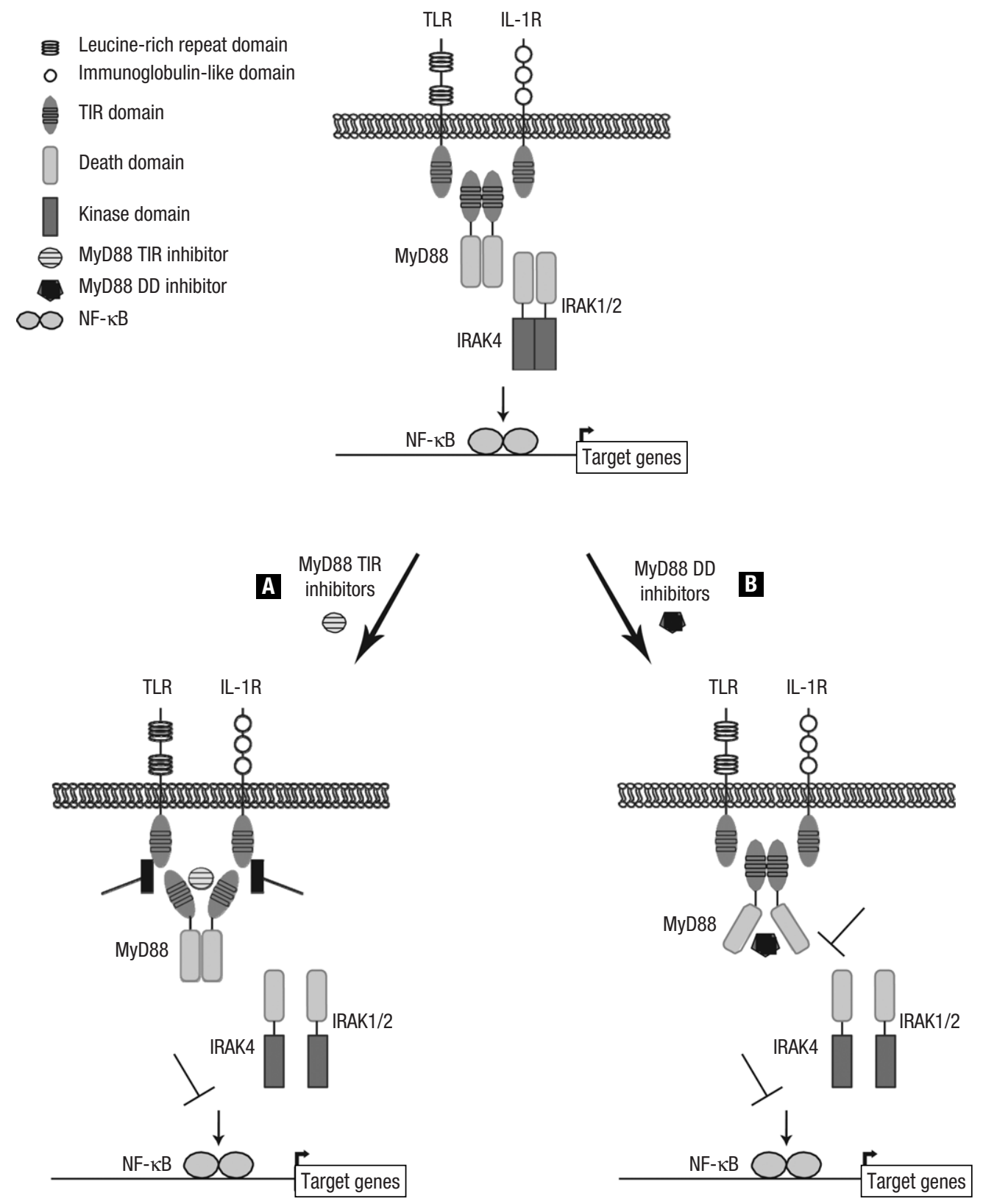

the TIR domain or DD, interaction of its TIR domain with TLR/ IL-1Rs, or recruitment of IRAKs by its DD (Figure 1).

To develop tools that interfere with the MyD88 function, we designed a multistep strategy. First, we analyzed the primary structure of the TIR and DD of MyD88 and of homologous proteins to identify residues that are evolutionary conserved, with specific attention to charged/polar residues that are likely to be involved in protein-protein interactions. Next, we performed 


\section{MyD88 Inhibition and Human Diseases}

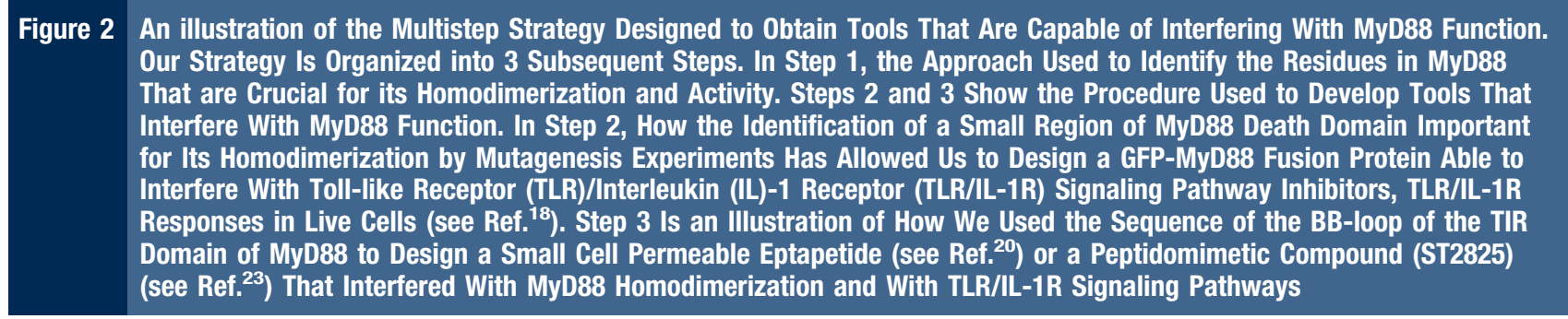

\section{Step 1}

Selection of residues to be mutated by alignment of

sequences of the TIR and Death domains of MyD88 with that of homologous proteins

Site-directed mutagenesis of selected residues by

substitution with alanine

Analysis of the effect of these mutations on

homodimerization of TIR and Death domains of MyD88
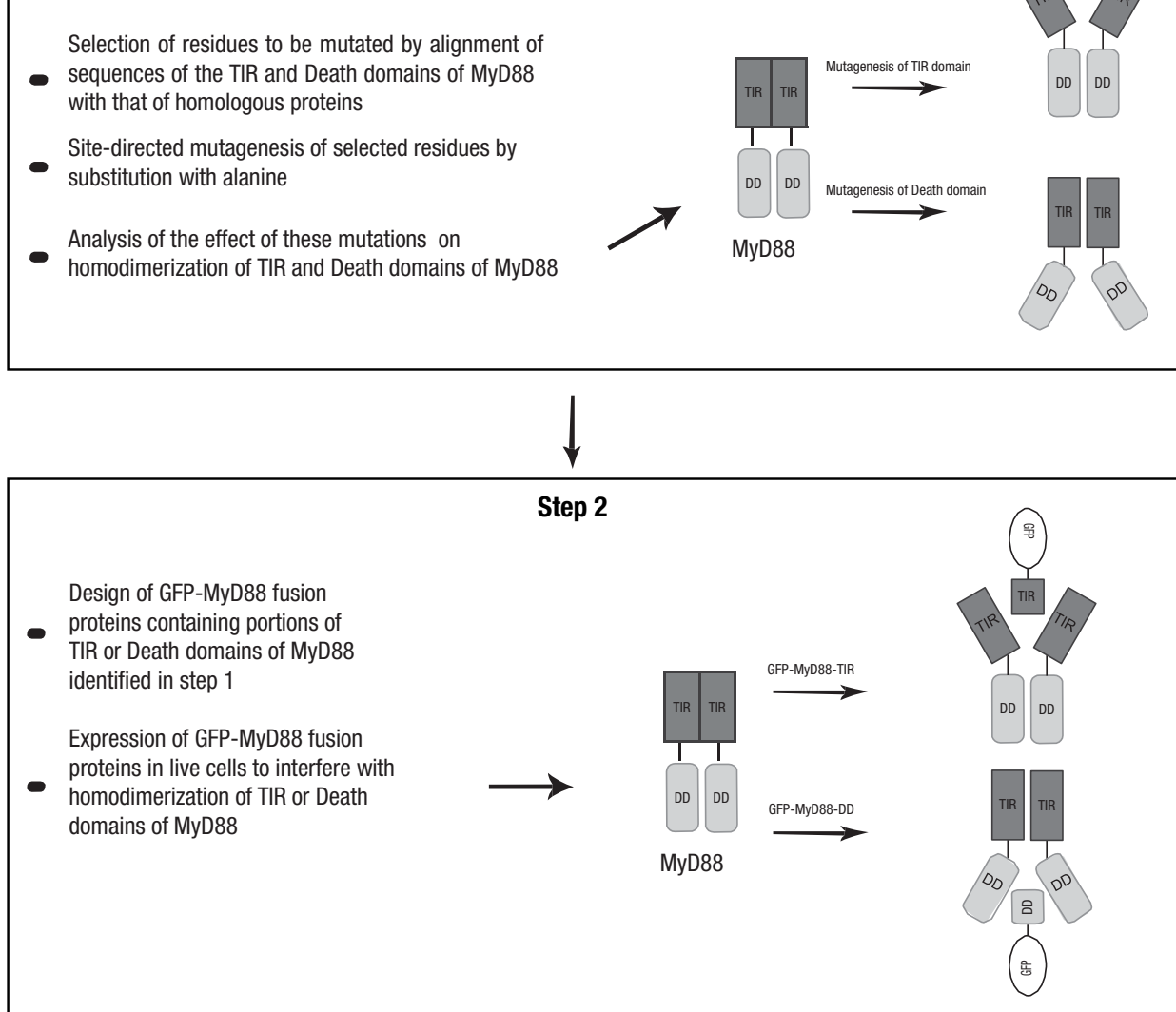

proteins containing portions of

TIR or Death domains of MyD88

proteins in live cells to interfere with

homodimerization of TIR or Death
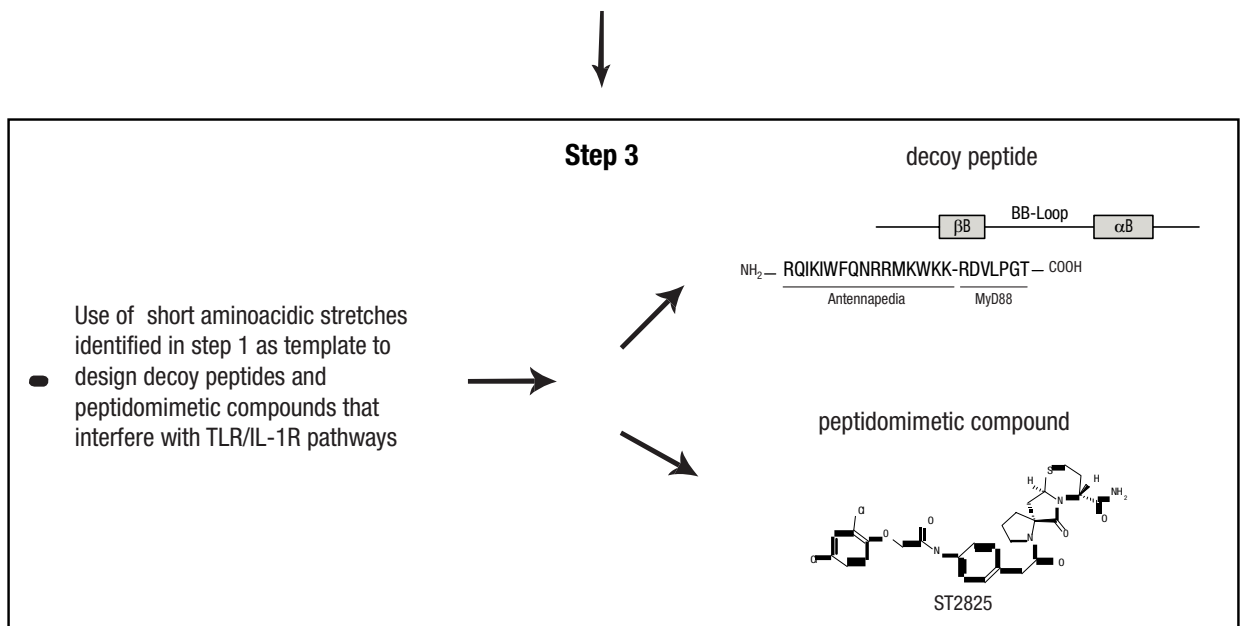
site-directed mutagenesis of such residues within the MyD88 TIR domain or DD and analyzed the ability of the mutant protein to homodimerize, to recruit IRAKs, and to activate NF- $\kappa$ B (step 1 in Figure 2). These features of the protein can be analyzed by dualtag coimmunoprecipitation assays (homodimerization and recruitment of IRAKs) and by reporter gene assays (NF- $\kappa \mathrm{B}$ activation). ${ }^{18}$

By following this strategy, we were able to identify 3 residues that are required for the interaction of MyD88 with IRAKs and for MyD88-dependent activation of NF- $\kappa \mathrm{B} .{ }^{18}$ Remarkably, mutations in one of these residues, glutamic acid 52, was also found in patients affected by recurrent infections, ${ }^{19}$ which thus validated our in vitro studies in a physiopathologic setting. Our studies also revealed that the small stretch of residues in the DD of MyD88, constituted by the $\alpha 1, \alpha 2, \alpha 3$ helices and by the first portion of the $\alpha 4$ helix, offers a surface of interaction between MyD88 and IRAKs. Step 2 in our strategy aims at interfering with MyD88 function by delivering portions of the molecule that can compete as a decoy with the endogenous MyD88 (Figure 2). We proved that expression of the above-mentioned small region of the MyD88 DD as fusion protein with a green fluorescent protein (GFP) moiety interfered with TLR/IL-1R responses. ${ }^{18}$ Thus, this short amino acidic stretch could serve as template to design decoy peptides and synthetic analogues (peptidomimetics) that might interfere with TLR/IL-1R pathways (step 3). We tested this possibility in another set of studies that targeted the TIR domain of MyD88, which also is required for homodimerization. Site-directed mutagenesis analyses identified a crucial stretch of residues in the BB-loop region, which connects the second $\beta$-strand with the second $\alpha$-helix of the TIR domain and is conserved among TLR/IL-1Rs. ${ }^{20}$ Mutation of these residues interfered with MyD88 dimerization and with downstream signaling events. ${ }^{21}$ Next, we synthesized a cell-permeable peptide by fusing the MyD88 aminoacidic sequence ArgAspValLeuProGlyThr (aa 196-202, RDVLPGT) to the antennapedia sequence that allows internalization in the cell ${ }^{20}$ (Figure 2) and showed that this MyD88 BB-loop decoy hepta-peptide blocked MyD88 homodimerization and reduced IL-1-dependent signaling in live cells. ${ }^{20}$

Natural peptides are not suitable drugs in vivo, due to their rapid clearance through proteolytic digestion. An alternative strategy is to develop synthetic molecules that closely mimic the original peptide. To this end, we developed and screened a series of synthetic molecules for their biologic activity toward MyD88 signaling. ${ }^{22}$ One such drug, ST2825 (Figure 2), was selected for its ability to interfere with MyD88 homodimerization and TLR9dependent signaling in vitro and in vivo. ${ }^{23}$ These studies proved that a rational design for the development of selective inhibitors of $\mathrm{MyD} 88$ is indeed possible.

\section{Application of MyD88 Inhibitors to Models of Human Diseases}

After our discoveries, the effect of the MyD88 decoy epta-peptide, the only commercially available MyD88-specific inhibitor, and of ST2825 have been analyzed in different models of human diseases. ${ }^{14,24,25}$ For instance, it was recently described that an oncogenic-activating mutation of MyD88 is responsible for the un- controlled proliferation of cells from patients affected by lymphomas and Waldenström's macroglobulinemia, a subgroup of immunoglobulin M-secreting non-Hodgkin lymphomas. In both studies, treatment of patient cells with the MyD88 decoy epta-peptide strongly inhibited oncogenic proliferation in vitro, ${ }^{13,14}$ which suggests that MyD88 inhibitors could be therapeutically suitable for these neoplastic diseases. This is particularly relevant for patients with Waldenström's macroglobulinemia, because a mutation of MyD88 was found in the large majority of patients affected by this neoplasia. ${ }^{14}$ The MyD88 peptide also was shown to downregulate inflammation in normal human knee articular chondrocytes stimulated with IL- $1 \beta,{ }^{24}$ which indicates its potential also in the treatment of chronic inflammatory diseases. In a similar vein, its synthetic analogue ST2825 was demonstrated to be effective in inhibiting the production of autoantibodies from patients with systemic lupus erythematosus, by blocking the generation of autoreactive plasma cells from memory B cells after TLR9 stimulation. ${ }^{25}$

It is foreseen that pharmacologic modulation of the TLR/IL-1R pathway might yield clinical benefits in the treatment of several human pathologies, including some forms of cancer. Thus, inhibitors of MyD88 function, such as those developed in our studies, are likely to represent a good therapeutic strategy to interfere with the molecular mechanisms of several human diseases triggered by aberrant activation of TLR/IL-1R-dependent signaling events.

\section{Acknowledgment}

This work was supported by grants from Sigma-Tau Industrie Farmaceutiche Riunite S.p.A., the Association for International Cancer Research, and the Fondazione Italiana Sclerosi Multipla.

\section{Disclosure}

The authors have stated that they have no conflicts of interest.

\section{References}

1. Kawai T, Akira S. Toll-like receptors and their crosstalk with other innate receptors in infection and immunity. Immunity 2011; 34:637-50.

2. Tang D, Kang R, Coyne CB, et al. PAMPs and DAMPs: signal 0s that spur autophagy and immunity. Immunol Rev 2012; 249:158-75.

3. Xu Y, Tao X, Shen B, et al. Structural basis for signal transduction by the toll/ interleukin-1 receptor domains. Nature 2000; 408:111-5.

4. O'Neill LA, Bowie AG. The family of five: TIR-domain-containing adaptors in toll-like receptor signaling. Nat Rev Immunol 2007; 7:353-64.

5. Hardiman G, Rock FL, Balasubramanian S, et al. Molecular characterization and modular analysis of human MyD88. Oncogene 1996; 13:2467-75.

6. Lin SC, Lo YC, Wu H. Helical assembly in the MyD88-IRAK4-IRAK2 complex in TLR/IL-1R signaling. Nature 2010; 465:885-90.

7. Brown J, Wang H, Hajishengallis GN, et al. TLR-signaling networks: an integration of adaptor molecules, kinases, and cross-talk. J Dent Res 2011; 90: 417-27.

8. Drexler SK, Foxwell BM. The role of toll-like receptors in chronic inflammation. Int J Biochem Cell Biol 2010; 42:506-18.

9. Merrell MA, Ilvesaro JM, Lehtonen N, et al. Toll-like receptor 9 agonists promote cellular invasion by increasing matrix metalloproteinase activity. Mol Cancer Res 2006; 4:437-47.

10. Fukata M, Chen A, Vamadevan AS, et al. Toll-like receptor-4 promotes the development of colitis-associated colorectal tumors. Gastroenterology 2007; 133: 1869-81.

11. Rakoff-Nahoum S, Medzhitov R. Regulation of spontaneous intestinal tumorigenesis through the adaptor protein MyD88. Science 2007; 317:124-7.

12. Swann JB, Vesely MD, Silva A, et al. Demonstration of inflammation-induced cancer and cancer immunoediting during primary tumorigenesis. Proc Natl Acad Sci US A 2008; 105:652-6.

13. Ngo VN, Young RM, Schmitz R, et al. Oncogenically active MYD88 mutations in human lymphoma. Nature 2011; 470:115-9.

14. Treon SP, Xu L, Yang G, et al. MYD88 L265P somatic mutation in Waldenström's macroglobulinemia. $N$ Engl J Med 2012; 367:826-33. 


\section{MyD88 Inhibition and Human Diseases}

15. Puente XS, Pinyol M, Quesada V, et al. Whole-genome sequencing identifies recurrent mutations in chronic lymphocytic leukaemia. Nature 2011; 475 : 101-15.

16. Loiarro M, Ruggiero V, Sette C. Targeting TLR/IL-1R signaling in human diseases. Mediators Inflamm 2010;2010:674363.

17. Connolly DJ, O'Neill LA. New developments in toll-like receptor targeted therapeutics. Curr Opin Pharmacol 2012; 12:510-8.

18. Loiarro M, Gallo G, Fantò N, et al. Identification of critical residues of the MyD88 death domain involved in the recruitment of downstream kinases. J Biol Chem 2009; 284:28093-103.

19. von Bernuth H, Picard C, Jin Z, et al. Pyogenic bacterial infections in humans with MyD88 deficiency. Science 2008; 321:691-6.

20. Loiarro M, Sette C, Gallo G, et al. Peptide-mediated interference of TIR domain dimerization in MyD88 inhibits interleukin-1-dependent activation of NF-(kappa)-B. J Biol Chem 2005; 280:15809-14.
21. Li C, Zienkiewicz J, Hawiger J. Interactive sites in the MyD88 toll/interleukin (IL) 1 receptor domain responsible for coupling to the IL1beta signaling pathway. J Biol Chem 2005; 280:26152-9.

22. Carminati P, Gallo G, Ruggiero V, et al. MyD88 homodimerization inhibitors. Patent Number WO2006067091.

23. Loiarro M, Capolunghi F, Fantò N, et al. Pivotal advance: inhibition of MyD88 dimerization and recruitment of IRAK1 and IRAK4 by a novel peptidomimetic compound. J Leukoc Biol 2007; 82:801-10.

24. Ahmad R, Sylvester J, Zafarullah M. MyD88, IRAK1 and TRAF6 knockdown in human chondrocytes inhibits interleukin-1-induced matrix metalloproteinase-13 gene expression and promoter activity by impairing MAP kinase activation. Cell Signal 2007; 19:2549-57.

25. Capolunghi F, Rosado MM, Cascioli S, et al. Pharmacological inhibition of TLR9 activation blocks autoantibody production in human B cells from SLE patients. Rheumatology (Oxford) 2010; 49:228119. 